\title{
Brunner Syndrome associated MAOA dysfunction in human induced dopaminergic neurons results in dysregulated NMDAR expression and increased network activity.
}

Y. Shi ${ }^{{ }^{*}}$, J.R. van Rhijn ${ }^{2^{*}}$, M. Bormann ${ }^{2}$, B. Mossink ${ }^{1}$, M. Frega ${ }^{1}$, M. Hakobjan ${ }^{1}$, S. Kittel-Schneider ${ }^{4}$, D.

Schubert $^{2}$, H. Brunner ${ }^{1}$, B. Franke ${ }^{1,3 \#}$, N. Nadif Kasri ${ }^{1,2 \#}$

*These authors contributed equally

"Shared final responsibility

${ }^{1}$ Department of Human Genetics, Donders Institute for Brain, Cognition and Behavior, Radboud

University Medical Center, Nijmegen, The Netherlands

${ }^{2}$ Department of Cognitive Neuroscience, Donders Institute for Brain, Cognition and Behavior, Radboud

University Medical Center, Nijmegen, The Netherlands

${ }^{3}$ Department of Psychiatry, Donders Institute for Brain, Cognition and Behavior, Radboud University

Medical Center, Nijmegen, The Netherlands

${ }^{4}$ Department of Psychiatry, Psychosomatic Medicine and Psychotherapy, Goethe-University,

Frankfurt, Germany 


\section{Abstract}

Monoamine oxidase $A$ (MAOA) is an enzyme that catalyzes the degradation of dopamine, noradrenaline, and serotonin. Regulation of monoamine neurotransmitter abundance through MAOA activity strongly affects motor control, emotion, and cognitive function. Mutations in MAOA cause Brunner Syndrome, which is characterized by impulsive aggressive behavior and mild intellectual disability (ID). The impaired MAOA activity in Brunner Syndrome patients results in bioamine aberration, but it is currently unknown how this affects neuronal function. MAOA is highly expressed in serotonergic and dopaminergic neurons, and dysfunction of both neurotransmission systems is associated with aggressive behavior in mice and humans. Research has so far mainly focused on the serotonergic system. Here, we generated human induced pluripotent stem cell-derived induced dopaminergic neurons (iDANs) from individuals with known MAOA mutations, to investigate MAOA-dependent effects on dopamine neuronal function in the context of Brunner Syndrome. We assessed iDAN lines from three patients and combined data from morphological analysis, gene expression, single-cell electrophysiology, and network analysis using micro-electrode arrays (MEAs). We observed mutation-dependent functional effects as well as overlapping changes in iDAN morphology. The most striking effect was a clear increase in N-methyl-D-aspartate (NMDA) receptor mRNA expression in all patient lines. A marked increase was also seen in coordinated network activity (network bursts) on the MEA in all patient lines, while single-cell intrinsic properties and spontaneous excitatory postsynaptic currents activity appeared normal. Together, our data indicate that dysfunction of MAOA leads to increased coordinated network activity in iDANs, possibly caused by increased synaptic NMDA receptor expression. 


\section{Introduction}

Monoamines, such as dopamine and serotonin, have an important role in brain function, and dysregulation of monoaminergic pathways is associated with several mental disorders including schizophrenia, autism spectrum disorder, and attention deficit/hyperactivity disorder $(A D H D)^{1}$. The abundance of monoamines is tightly regulated by monoamine oxidases (MAOs), which catabolize the monoaminergic neurotransmitters ${ }^{2}$. Disruption of MAO activity can thus have profound consequences on normal brain function ${ }^{3}$. Brunner Syndrome is a neurodevelopmental disorder characterized by hemizygous mutation of the X-linked monoamine oxidase, and was first described in a large Dutch kindred with non-dysmorphic borderline intellectual disability (ID) and prominent impulsive aggressive behavior $^{4,5}$. Recently, three more families have been reported in which affected individuals have Brunner Syndrome and carry either nonsense or missense mutations of $M A O A^{6,7}$, which strengthens the association between MAOA dysfunction and Brunner Syndrome.

Changes in monoaminergic regulation, especially of serotonin and dopamine, have also been associated with aggressive behavior in animal models, both in wild-type animals ${ }^{8-11}$ as well as in genetic models for neurodevelopmental disorders ${ }^{12}$. Transgenic mouse models of Maoa have allowed the exploration of mechanistic associations between monoamine regulation and aggressive behavior. Hemizygous Maoa mutant mice show abnormally high levels of aggressive behavior and disturbed monoamine metabolism, similar to the patient situation ${ }^{13-15}$. Maoa-deficient mice also display alterations in brain development, with aberrant organization of the primary somatosensory cortex ${ }^{13}$ and increased dendritic arborization of pyramidal neurons in the orbitofrontal cortex ${ }^{15}$. Furthermore, Maoa has been implicated in the regulation of synaptic neurotransmitter receptors, as Maoa knockout mice show increased $\mathrm{N}$-methyl-D-aspartate (NMDA) receptor subunit expression in the prefrontal cortex ${ }^{16}$. Taken together, these data suggest that Maoa can influence both structural and functional aspects of neurodevelopment.

MAOA is expressed in different neuronal as well as glial cell types in the brain ${ }^{17}$. However, not all of these cell types are thought to be associated with the behavioral phenotypes observed in MAOA mutation carriers. So far, particular emphasis has been given to the serotonergic system in MAOA research, but MAOA is also abundantly expressed in dopaminergic neurons ${ }^{18,19}$, and aggressive behavior is highly associated with aberrant dopamine pathway function ${ }^{1,20}$. The current advances in the generation of human pluripotent stem cell (iPSC)-induced neurons (iNeurons) enable us to 
generate a homogenous culture of a single neuronal cell type. We therefore generated iPSC-derived induced dopaminergic neurons (iDANs), which offer a unique opportunity to investigate the cellular and molecular mechanisms specifically affected by MAOA dysfunction in dopaminergic neurons. We generated iDANs from three independent male individuals with unique MAOA mutations and from two independent male controls. Combining data from morphological analysis, gene expression, single-cell electrophysiology, and network analysis using micro electrode arrays (MEAs), we suggest that alterations in dopaminergic neuron development and function may also be relevant to behavioural and cognitive dysfunction in Brunner Syndrome.

\section{Results}

\section{Generation of control and Brunner Syndrome patient-derived iDANs}

Using a protocol adapted from ${ }^{21}$, we successfully differentiated iPSCs into iDANs after 55 days in vitro (DIV55, Figure 1a). We generated iDANs from two independent control (control-1 and control-2) and three independent patient iPSC lines (Patient-NE8, c.886C>T, p.Q296*; Patient-ME8, c.797_798delinsTT, p.C266F; Patient-ME2, c.133C>T, p.R45W; Figure 1b, for extended information see Supplementary Table S2). Neuronal identity was confirmed by microtubule-associated protein 2 (MAP2) expression and iDAN identity by expression of tyrosine hydroxylase (TH) (Figure 1c). Cell type specificity was comparable between lines, with more than $90 \%$ of MAP2-expressing neurons staining positive for TH (Figure 1d). Interestingly, mRNA levels of MAOA were only altered compared to the healthy controls in the Patient-NE8 line, which has a nonsense mutation (Figure 1e). The observed reduction might have been caused by nonsense-mediated mRNA decay, as shown earlier in human fibroblasts with the same mutation ${ }^{25}$. We used these five lines in subsequent experiments to investigate how MAOA dysfunction affects iDAN development and function.

\section{Mutation-specific effects of MAOA-disruption on iDAN morphology}

Dendritic properties of neurons in the orbitofrontal cortex are known to be altered in Maoa hemizygous knockout mice ${ }^{15}$. To assess whether MAOA dysfunction also affects the morphology of iDANs, we compared dendritic properties and soma size of control and patient iDANs by quantitative morphometric analysis of the somatodendritic compartment. ME8, NE8 and ME2 patient lines revealed mutation-dependent effects on iDAN morphology. Only iDANs with ME8 mutation showed increases in 
soma size (Figure 2b) and dendrite complexity (Figure 2c,d,e,f). Additionally, iDANs with the ME8 and ME2 mutations showed a significant increase in total covered surface (Convex Hull analysis, Figure 2g), whilst iDANs with the NE8 mutation showed no differences in neuronal complexity. Next, we investigated if these differences in morphology could affect the number of excitatory synapses present on iDANs, since changes in neuronal complexity ${ }^{26,27}$ and synapse density ${ }^{28}$ are widely associated with neurodevelopmental disorders. Indeed, synapse density was significantly decreased in iDANs from all patients compared to those from control-1 (Figure $2 \mathbf{h}, \mathbf{i}$ ). This suggests that differences in synapse density might be caused by a molecular mechanism affected across mutations, whereas the drastic difference in iDAN dendritic morphology might be mutation-specific.

\section{MAOA dysfunction leads to increased NMDAR expression in iDANs}

The alterations in morphology and synapse density in MAOA hemizygous mutation iDANs prompted us to investigate whether gene expression of synapse-specific genes might be affected by MAOA dysfunction. In excitatory neurons, both a-amino-3-hydroxy-5-methyl-4-isoxazolepropionic acid receptors (AMPARs) and N-methyl D-aspartate receptors (NMDARs) are especially relevant for glutamatergic synaptic neurotransmission. Both AMPARs and NMDARs are heteromeric ion channels comprised of four subunits, and the ion channel composition conveys differences in ion selectivity as well as opening and closing kinetics. We therefore investigated the expression of the four main isoforms of the AMPA receptor subunits GluA1, 2, 3 and 4 (encoded by GRIA1, 2, 3, and 4, respectively) as well as the expression of the NR1, NR2A, and NR2B subunits (encoded by GRIN1, $2 A$ and $2 B$, respectively) of the NMDA receptor.

When we compared control iDANs at DIV73 to our patient-derived iDANs, no difference in AMPAR subunit expression was seen (Figure S3a-d). This suggests AMPAR-mediated neurotransmission is not affected in dopaminergic neurons by mutations in $M A O A$. Following evidence from Maoa knockout mice showing that NMDAR expression is altered in the prefrontal cortex ${ }^{16}$, we also investigated NMDAR in our patient-derived iDANs compared to controls. No difference in gene expression was found for GRIN1 (Figure 3a), but increased expression was seen for both GRIN2A and GRIN2B in the patient-derived iDANs (Figure 3b,c), similar to the earlier observations in Maoa knockout mice ${ }^{16}$. Together, this data suggests that excitatory neurotransmitter receptor expression at the synapse is altered by MAOA dysfunction. 
Intrinsic properties and AMPAR-mediated excitatory activity are not affected by MAOA mutation

As NMDAR expression is tightly linked to burst firing in dopaminergic neurons ${ }^{29}$ and since differences in neuronal morphology and synapse density, as we observed them, can have drastic consequences for neuronal activity, we next explored if physiological activity is altered by MAOA dysfunction in iDANs. First, we explored whether MAOA dysfunction affects synapse physiology through altered excitatory neuron function using whole-cell patch clamp. Comparing the intrinsic properties of the control lines, we found no significant differences between control-1 and control-2 (Figure 4a-d) and pooled the data for comparison with the patient-derived lines. No differences in intrinsic properties were observed between the pooled data of the control lines and the three patient lines (Figure 4e-h). To assess whether AMPAR-mediated inputs are affected by MAOA dysfunction, we measured spontaneous excitatory postsynaptic currents (sEPSCs). Again, we first compared control lines and found no differences in sEPSC amplitude or frequency (Figure 4i-k). Comparison of the pooled control data to data from the individual patients revealed that AMPAR-mediated excitatory activity was not

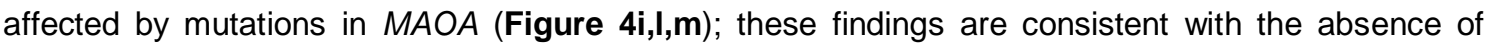
differences in the expression of GluA subunit mRNA between patients and controls.

\section{MAOA dysfunction leads to increased iDAN network activity recorded by MEA}

Since we found no differences in intrinsic properties and spontaneous AMPAR-driven activity at the single cell level, we next explored whether the increased NMDAR mRNA expression in MAOA-mutant iDAN lines leads to changes in neuronal activity at the network level. Therefore, we measured network activity of iDANs with MEA. Spontaneous random activity was clearly visible for all five iDAN cell lines at DIV73 (Figure 5a), and robustly higher mean firing rates were seen for all patient lines compared to the activity in both control lines (Figure 5b,c). Furthermore, whilst the control lines exhibited almost no coordinated network bursts, these were clearly visible in each of the patient-derived lines (Figure 5a, colored blocks in traces), and their frequency was significantly higher (Figure $\mathbf{5 d , e ) . ~ T h i s ~ s u g g e s t s ~}$ that both missense and nonsense mutations of MAOA lead to increased activity in excitatory dopaminergic neurons, possibly through upregulation of NMDAR-driven network excitation. 


\section{Discussion}

Dysfunction of MAOA in humans results in Brunner Syndrome. Here, we investigated Brunner Syndrome in a human iPSC-derived neuronal model for the first time, choosing dopaminergic neurons as our model. We generated two control and three patient lines and compared morphology, expression of synaptic genes, and neuronal physiology at the single-cell and network level. Confirming findings obtained in a Maoa knockout mouse model ${ }^{15,16}$, we found neuronal complexity to be higher in dopaminergic neurons derived from humans carrying $M A O A$ mutations. Our work further extends current knowledge by showing that such differences are mutation-dependent, as the p.C266F mutation (ME8) showed the strongest differences in iDAN morphology, whereas in the p.Q296* (NE8) line such alterations were less severe, and no differences were seen in the p.R45W (ME2) line. This suggests that different mutations might uniquely affect the function of the MAOA, as has been hypothesized in in vitro studies of MAOA function ${ }^{30}$. In addition to the mutation-specific effects on neuronal morphology, we observed several differences in neuronal function shared amongst all mutations. Firstly, we observed an increase in NMDAR mRNA expression, whilst AMPAR mRNA levels were unchanged. While intrinsic properties and spontaneous activity of single cells were unaffected, neuronal activity at the network level was robustly higher in all patient-derived lines compared to control iDANs.

The differences in spontaneous activity and in NMDAR expression could be related. Firstly, it has been shown that in Maoa knockout mice NMDAR expression is increased, which affects NMDARdependent currents in prefrontal cortex ex vivo slice preparations of these mice ${ }^{16}$. Furthermore, increased NMDAR-expression is able to enhance spontaneous neuronal activity during development $^{31}$, and dysfunctional enhancement of NMDAR signaling has been described in other neurodevelopmental disorders ${ }^{32}$. However, how MAOA mutation and increased NMDAR expression are related on a mechanistic level needs to be further explored.

Synapse density was found reduced across patient-derived lines. This could be seen a compensatory mechanism in the case of the higher neuronal complexity in the ME8 patient line, but this cannot explain the reduced synapse density in the other patient lines. The expression and localization of NMDARs has been shown to regulate dendritic arborization ${ }^{33-35}$. It is therefore conceivable that the 
increased dendrite complexity and the changes in synapse density in the MAOA-mutant lines can be partially explained by the increased NMDAR expression.

The individuals we investigated in our study all carry rare mutations, which lead to either complete loss or reduced activity of the MAOA enzyme. The MAOA promoter also can exhibit different levels of transcriptional activity ${ }^{39}$ through a common variable number of tandem repeats (VNTR) polymorphism in the MAOA regulatory region, located $1.2 \mathrm{~kb}$ upstream of the transcription initiation site. Low activity alleles of $M A O A$ that show similar transcriptional activity as the missense mutations present in Brunner Syndrome patients have been associated with the anti-social behavior in individuals subjected to childhood maltreatment ${ }^{40,41}$. Furthermore, an association has been observed between the severity of ASD features and the presence of $M A O A$ low activity alleles ${ }^{42,43}$, which suggests that $M A O A$ also plays a role in ASD pathology ${ }^{38}$. Lastly, impulsive aggressive behavior has been described as a feature of other, genetically complex neurodevelopmental disorders such as $\mathrm{ADHD}^{44}$ and $\mathrm{ASD}^{45}$, in which monoaminergic signaling seems to be involved but the association with MAOA allele activity has not yet been extensively explored. Interestingly, using magnetic resonance imaging (MRI) in a large group of healthy individuals, we recently showed differences in structural and functional network architecture between carriers of low and high activity MAOA alleles, where those with low activity alleles had more extensive networks. Investigation of MAOA function in neurons derived from individuals with low or high activity MAOA alleles might thus help us further understand the molecular mechanisms regulated by MAOA and how MAOA activity might influence impulsive aggressive behavior in the general population.

Lastly, MAOA dysfunction does not exclusively affect the dopaminergic system, as it also results in increased serotonin levels in both human and mice $5,6,7,13,15,46$. Additionally, the serotonergic pathway is, similar to the dopaminergic pathway, associated with aggression and impulsivity ${ }^{47}$. Previously, it was suggested the increased serotonin levels are an indirect effect of reduced MAOA activity, as MAOA was shown not to be expressed in serotonergic neurons. However, recent single cell RNAseq profiling revealed MAOA is expressed both in neural progenitor cells and monoaminergic neurons, including both dopaminergic and serotonergic neurons ${ }^{19,48}$.

This study should be viewed in the context of its strengths and limitations. This is the first study on Brunner Syndrome investigating the molecular, cellular, and circuit alterations underlying the 
phenotype of the patients in iPSC-derived homogeneous neuronal cultures. The wide range of methods used and the integration of findings allowed us to validate results of animal models and extend our insight into the biological underpinnings of Brunner Syndrome further.

In conclusion, we established iDANs as a pure iPSC-derived human neuron culture that can be used to investigate disorders, which specifically affect monoaminergic pathways. Our data on MAOA dysfunction using this tool suggest that dopaminergic neurons may also be altered in humans carrying MAOA mutations, extending research focused on the serotonergic system. We confirm work performed in rodent models and non-neuronal transgenic models and extend such knowledge further by identifying both mutation-specific as well as general effects of MAOA dysfunction. Our findings of altered network activity may also link to human MRI studies showing altered structural and functional networks in carriers of a functional polymorphism in the MAOA gene. Beyond investigation of the biological underpinnings of neurodevelopmental disorders like Brunner Syndrome, the infrastructure we built may also provide a platform to investigate potential (individualized) therapeutic interventions for disorders in which monoamine regulation is disrupted. 


\section{Materials and Methods}

\section{Cell Culture of iPSCs}

The iPSC line control-1 was derived from a dermal fibroblast biopsy of a male healthy volunteer. iPSC line control-2 was obtained from ${ }^{50}$ and was generated from a healthy male donor using an episomal reprogramming $^{51}$. The iPSC lines patient-NE8, patient-ME8 and patient-ME2 that carry MAOA mutations were derived from dermal fibroblast biopsies of male patients described previously ${ }^{4-7}$. The fibroblasts were reprogrammed by introducing four Yamanaka factors: Sox2, Klf4, Oct4, c-Myc via a lentivirus-based method. All iPSC reprogramming was done by the Radboudumc Stem Cell Technology Center (SCTC). iPSC pluripotency was characterized by immunocytochemistry and qPCR (supplemental figure S1). The iPSCs were maintained in Essential 8 complete medium with $100 \mu \mathrm{g} / \mathrm{ml}$ penicillin/streptomycin or $100 \mu \mathrm{g} / \mathrm{ml}$ Primocin (InvivoGen) on vitronectin (VTN-N)-coated cell culture plates (Corning) at $37^{\circ} \mathrm{C} / 5 \% \mathrm{CO} 2$. Cell colonies were passaged by $0.5 \mathrm{mM}$ ultrapure EDTA treatment for 2 or 3 minutes in DPBS without calcium and magnesium. Unless indicated otherwise, all the reagents were bought from Thermo Fisher Scientific Inc.

\section{Differentiation of iPSCs into dopaminergic neurons}

The iPSC colonies were split into single cells by Accutase treatment for $5 \mathrm{~min}$ at $37^{\circ} \mathrm{C}$ two days before starting the differentiation, and $1-2 \times 10^{4} / \mathrm{cm}^{2}$ cells were replated on a vitronectin-coated plate in Essential 8 complete medium supplemented with either $2 \mathrm{uM}$ thiazovivin(Sigma) or $1 \mathrm{X}$ RevitaCell Supplement. The differentiation protocol of dopaminergic neurons from iPSCs was modified from ${ }^{21}$. From day 0 to day 5, the E8 complete medium was replaced with Knockout DMEM, supplemented with 15\% Knockout Serum Replacement, 1x GlutaMAX, 100U/mL Penicillin-Streptomycin and 1x MEM-NEAA. The $0.1 \mathrm{mM} \beta$-Mercaptoethanol was added freshly before changing the medium. From day 6 to day 9 , the Knockout DMEM medium was gradually replaced by $25 \%, 50 \%, 75 \%$ and $100 \%$ N2 medium (DMEM/F12 with $1 \times \mathrm{N}-2$ supplement) respectively. The iDANs were cultured in N2 medium until day 11 . From day 12 on, the iDANs were cultured in neurobasal medium supplemented with 1x B27 and 1x GlutaMAX and half medium was refreshed every two days. During the differentiation and lineage specification, different combinations of small molecules and/or growth factors were added at different time points: $10 \mu \mathrm{M} L D N-193189$ (Stemgent Inc.) from day 1 to day 11; $10 \mu \mathrm{M}$ SB431542 (Stemgent Inc.); from day1 to day 5; $2 \mu \mathrm{M}$ Purmorphamine (Stemgent Inc.); from day 2 to day $11 ; 100 \mathrm{ng} / \mathrm{mL}$ recombinant human fibroblast growth factor 8 a (FGF-8a, R\&D system) from 
day 2 to day 11; $3 \mu \mathrm{M}$ CHIR99021 (Stemgent Inc.); from day 3 to day 12; 100ng/ml recombinant human sonic hedgehog (C24II, SHH, R\&D system); From day 12 to the end of the differentiation, $20 \mathrm{ng} / \mathrm{ml}$ recombinant brain derived neurotrophic factor (BDNF, Peprotech), 20ng/ml recombinant glialderived neurotrophic factor (GDNF, Peprotech), 0.5mM adenosine 3',5'-cyclic monophosphate (cAMP, Enzo Life Science), 2ng/ml transforming growth factor beta 3 (TGFß3, Millipore), $200 \mu \mathrm{M}$ ascorbid acid (AA, Sigma) and 10nM $y$-secretase inhibitor IX (DAPT, Millipore, Calbiochem) were added into the medium. The cells were passaged only when they were $100 \%$ confluent using accutase. At day 20 , the iDANs were split into single cells and $1-2 \times 10^{4} / \mathrm{cm}^{2}$ cells were replated on a poly-L-ornithine

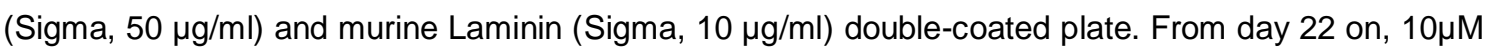
DAPT was used to promote iDAN maturation. Rat astrocytes (prepared and plated according to ${ }^{22}$ ) were cocultured with iDANs from day 24 to further promote maturation.

\section{Gene expression analysis}

To analyze the gene expression profile, the RNA was isolated from iPSCs and differentiated neurons with the RNeasy Mini Kit (Qiagen) according to the manufacturer's instructions. 0,5-1ug RNA was retro-transcribed into cDNA by the iScript cDNA Synthesis Kit (Bio-Rad Laboratories, Inc) according to the manufacturer's instructions. The gene expression of iPSCs and differentiated iDANs was measured by quantitative real-time PCR (qRT-PCR) which was performed with the Applied Biosystems 7500 Fast Real-Time PCR System using Power SYBR Green Master Mix (Applied Biosystems) and qRT-PCR primers (listed in Supplementary table S1). Beta-2-Microglobulin (B2M) was used as reference gene. The $\mathrm{Ct}$ value of each target gene was normalized against the $\mathrm{Ct}$ value of the reference gene $\left[\Delta \mathrm{Ct}=[\mathrm{Ct}(\operatorname{target})-\mathrm{Ct}(\mathrm{B} 2 \mathrm{M})]\right.$. The relative expression was calculated as $2^{-\Delta \Delta \mathrm{Ct}}$ and represented as fold change of gene expression when compared to corresponding control conditions $[2$ $\left.\Delta \Delta \mathrm{Ct}=2^{\Delta \mathrm{Ct}(\text { target) }-\Delta \mathrm{Ct}(\text { control) }}\right]$.

\section{Immunocytochemistry}

The cells were fixed with $4 \%$ paraformaldehyde $/ 4 \%$ sucrose(v/v)(Sigma) in PBS for $15 \mathrm{~min}$ at room temperature (RT) before the immunocytochemistry experiment. The non-specific binding of antibodies was avoided by incubating the cells with $5 \%$ normal goat serum(Thermo Fisher Scientific)/ $0.4 \%$ Triton X-100(Sigma)/1\% Glycine(Sigma) in PBS (blocking solution) at RT for one hour. The primary and secondary antibodies which were also diluted in the blocking solution and applied overnight at $4^{\circ} \mathrm{C}$ or 
$1 \mathrm{~h}$ at RT. Cell nuclei were stained with Hoechst $(1: 10000)$ and the coverslips were mounted with DAKO fluoromount medium (Agilent). The primary antibodies were used: mouse anti-MAP2 (1:1000; Sigma M4403); guinea pig anti-MAP2 (1:1000; Synaptic Systems 188004); guinea pig anti-synapsin 1/2 (1:1000; Synaptic Systems 106004); mouse anti-TH (1:200; Sigma TH-16). Secondary antibodies used were: goat anti-guinea pig Alexa Fluor 568 (1:1000, Invitrogen A-11075); goat anti-rabbit Alexa Fluor 488 (1:1000, Invitrogen A-11034); goat anti-mouse Alexa Fluor 488 (1:1000, Invitrogen A11029); goat anti-mouse Alexa Fluor 568 (1:1000, Invitrogen A-11031).

\section{Microelectrode array and data analysis}

The neuronal network activity was measured using 6-Well Microelectrode arrays devices (Multichannel Systems, MCS GmbH, Reutlingen, Germany) consisted of $60 \mathrm{TiN}$ (titanium nitride)/SiN(silicon nitride) planar round electrodes (30 $\mu \mathrm{m}$ diameter; $200 \mu \mathrm{m}$ center-to-center interelectrode distance) divided into 6 separated wells. Each well was characterized by 9 recording electrodes, arranged in a $3 \times 3$ square grid, and a ground electrode. The activity of all cultures was recorded for $20 \mathrm{~min}$ by means of the MEA60 System (MCS GmbH, Reutlingen, Germany) with a high pass filter (Butterworth, $100 \mathrm{~Hz}$ cutoff frequency). During the recording, the temperature was maintained at $37^{\circ} \mathrm{C}$ by means of a controlled thermostat (MCS GmbH, Reutlingen, Germany). Additionally, evaporation and $\mathrm{pH}$ changes of the medium was prevented by continuous perfusion with carbogen $\left(95 \% \mathrm{O}_{2}, 5 \% \mathrm{CO}_{2}\right)$ inside a humidified compartment with an open connection to the MEA. After 1200x amplification, signals were sampled at $10 \mathrm{kHz}$ and acquired through MC-Rack software (MCS GmbH, Reutlingen, Germany).

Data analysis was performed off-line by using a custom software developed in MATLAB (The Mathworks, Natick, MA, USA): SPYCODE ${ }^{22,52}$, which collects a series of tools for processing multichannel neural recordings. Spikes and bursts were detected by using the Precise Timing Spike Detection algorithm (PTSD) ${ }^{53}$ and the Burst Detection algorithm ${ }^{54}$. The mean firing rate (spikes/s) of the network was computed by averaging the firing rate of each channel with all the active electrodes of the MEA. The burst was computed as at least 5 spikes in a burst with a maximal inter-spike-interval of 80 milliseconds. The network burst was defined as synchronized bursts that occurs in $>50 \%$ of the active channels. And the network burst rate (burst/min) was calculated as the amount of network bursts per minute.

\section{Single-cell electrophysiology}


Experiments were conducted on DIV55 iDANs. The coverslip with neurons was transferred the to a recording chamber continuously perfused with oxygenated $\left(95 \% \mathrm{O}_{2} / 5 \% \mathrm{CO}_{2}\right)$ and heated $\left(32^{\circ} \mathrm{C}\right)$ recording ACSF containing (in mM): $124 \mathrm{NaCl}, 3 \mathrm{KCl}, 1.25 \mathrm{NaH}_{2} \mathrm{PO}_{4}, 2 \mathrm{CaCl}_{2}, 1 \mathrm{MgCl}_{2}, 26 \mathrm{NaHCO}_{3}$, 10 Glucose. Patch pipettes (5.5-7.5 M $)$ ) were made from borosilicate glass capillaries and filled with intracellular recording solution containing (in $\mathrm{mM}$ ): $130 \mathrm{~K}$-Gluconate, $5 \mathrm{KCl}, 10 \mathrm{HEPES}, 2.5 \mathrm{MgCl}_{2}, 4$ $\mathrm{Na}_{2}$-ATP, $0.4 \mathrm{Na}_{3}$-ATP, 10 Na-phosphocreatine, 0.6 EGTA (adjusted to pH 7.25 and osmolarity 290 mosmol). Activity was recorded using a Digidata 1440A digitizer and a Multiclamp 700B amplifier (Molecular Devices). Sampling rate was set at $20 \mathrm{KHz}$ and a lowpass $1 \mathrm{KHz}$ filter was used during recording. Series resistance was monitored on-line and cells were discarded if series resistance increased above 1:10 of membrane resistance.

Spontaneous postsynaptic currents

sEPSCs were recorded in drug free recording medium at a holding potential of $-60 \mathrm{mV}$.

Intrinsic properties

Intrinsic properties were recorded in drug free medium. Current-voltage responses were recorded by 1 second stepwise injection of current in $5 \mathrm{pA}$ increments. Current injection continued until clear action potentials were visible or until a maximum current of 100 pA was injected. Intrinsic properties were calculated off-line using Clampfit 10.7 software (Molecular Devices, San Jose, CA). Statistical comparison was conducted in PRISM (Graphpad PRISM 7.00, Graphpad Software, San Diego, CA).

\section{Neuronal morphology reconstruction and data analysis}

Wide field fluorescent images of the fixed and MAP2-labelled iPSC derived dopaminergic neurons were taken at 20x magnification using ApoTome microscopy (Zeiss Axio Imager Z1/Z2). The images were stitched using Fiji 2017 software with the stitching plugin and digitally reconstructed using Neurolucida 360 (Version 2017.01.4, Microbrightfield Bioscience, Williston, USA) to create an overlay drawing of the somatodendritic morphology. Only the neurons that have at least two primary dendrites and at least one dendritic branch point were selected for the reconstruction.

The quantitative analysis of the somatodendritic organization of the dopaminergic neurons was conducted using Neurolucida 360 Explorer (Version 2017.02.7, Microbrightfield Bioscience, Williston, USA). For the somatodendritic analysis, the soma area, number of dendritic nodes, dendritic length, quantity of primary dendrites, as well as the covered surface by convex hull $2 \mathrm{D}$ analysis were determined. Furthermore, Sholl analysis was performed to investigate the dendritic complexity ${ }^{55}$. The 
dendritic length of the neurons was measured within a series of concentric circles at $25 \mu \mathrm{m}$ interval from the soma. All the morphological data were acquired and analyzed blindly to the genotype of the neurons. For the somatodendritic properties and Sholl analysis, significance was determined using IBM SPSS Statistics (version 24.0, IBM, Armonk, USA) to run Wilks' Lambda multivariate analysis of variance (MANOVA) followed by the Bonferroni post-hoc.

\section{Statistical analysis}

Statistical analysis of the data was performed with GraphPad Prism 5.0.3 (GraphPad Software, Inc, USA). And the distribution of data is expressed as mean \pm SEM. Mann-Whitney $U$ test, unpaired Student's T test or one-way ANOVA with Tukey's correction for multiple-comparisons was used for statistical analysis. $p<0.05$ was considered significant. 

dopamine. Front Cell Neurosci 7, 260, doi:10.3389/fncel.2013.00260 (2013) Godar, S. C., Fite, P. J., McFarlin, K. M. \& Bortolato, M. The role of monoamine oxidase A in aggression: Current translational developments and future challenges. Prog Neuropsychopharmacol Biol Psychiatry 69, 90-100, doi:10.1016/j.pnpbp.2016.01.001 (2016). Bortolato, M., Chen, K. \& Shih, J. C. Monoamine oxidase inactivation: from pathophysiology to therapeutics. Adv Drug Deliv Rev 60, 1527-1533, doi:10.1016/j.addr.2008.06.002 (2008). Brunner, H. G., Nelen, M., Breakefield, X. O., Ropers, H. H. \& van Oost, B. A. Abnormal behavior associated with a point mutation in the structural gene for monoamine oxidase $A$. Science 262, 578-580 (1993). Brunner, H. G. et al. X-linked borderline mental retardation with prominent behavioral disturbance: phenotype, genetic localization, and evidence for disturbed monoamine metabolism. Am J Hum Genet 52, 1032-1039 (1993).

Piton, A. et al. 20 ans apres: a second mutation in MAOA identified by targeted highthroughput sequencing in a family with altered behavior and cognition. Eur J Hum Genet 22, 776-783, doi:10.1038/ejhg.2013.243 (2014).

7 Palmer, E. E. et al. New insights into Brunner syndrome and potential for targeted therapy. Clinical Genetics 89, 120-127, doi:10.1111/cge.12589 (2016).

8 van Erp, A. M. \& Miczek, K. A. Aggressive behavior, increased accumbal dopamine, and decreased cortical serotonin in rats. J Neurosci 20, 9320-9325 (2000).

9 Ferrari, P. F., van Erp, A. M., Tornatzky, W. \& Miczek, K. A. Accumbal dopamine and serotonin in anticipation of the next aggressive episode in rats. Eur J Neurosci 17, 371-378 (2003).

10 Alekseyenko, O. V., Chan, Y. B., Li, R. \& Kravitz, E. A. Single dopaminergic neurons that modulate aggression in Drosophila. Proceedings of the National Academy of Sciences of the United States of America 110, 6151-6156, doi:10.1073/pnas.1303446110 (2013).

11 Couppis $\mathrm{MH}$, Kennedy $\mathrm{CH}$. The rewarding effect of aggression is reduced by nucleus accumbens dopamine receptor antagonism in mice. Psychopharmacology. 197:449456(2008).

12 Rodriguiz, R. M., Chu, R., Caron, M. G. \& Wetsel, W. C. Aberrant responses in social interaction of dopamine transporter knockout mice. Behav Brain Res 148, 185-198 (2004).

13 Cases, 0 . et al. Aggressive behavior and altered amounts of brain serotonin and norepinephrine in mice lacking MAOA. Science 268, 1763-1766 (1995).

14 Scott, A. L., Bortolato, M., Chen, K. \& Shih, J. C. Novel monoamine oxidase A knock out mice with human-like spontaneous mutation. NeuroReport 19, 739-743, doi:10.1097/WNR.0b013e3282fd6e88 (2008).

15 Bortolato, M. et al. Social Deficits and Perseverative Behaviors, but not Overt Aggression, in MAO-A Hypomorphic Mice. Neuropsychopharmacology 36, 2674-2688, doi:http://www.nature.com/npp/journal/v36/n13/suppinfo/npp2011157s1.html (2011).

16 Bortolato, M. et al. NMDARs Mediate the Role of Monoamine Oxidase A in Pathological Aggression. Journal of Neuroscience 32, 8574-8582, doi:10.1523/Jneurosci.0225-12.2012 (2012).

17 Luque, J. M., Kwan, S. W., Abell, C. W., Da Prada, M. \& Richards, J. G. Cellular expression of mRNAs encoding monoamine oxidases $A$ and $B$ in the rat central nervous system. $J$ Comp Neurol 363, 665-680, doi:10.1002/cne.903630410 (1995).

18 Nagatsu, T. Progress in monoamine oxidase (MAO) research in relation to genetic engineering. Neurotoxicology 25, 11-20, doi:10.1016/S0161-813X(03)00085-8 (2004).

19 La Manno, G. et al. Molecular Diversity of Midbrain Development in Mouse, Human, and Stem Cells. Cell 167, 566-580 e519, doi:10.1016/j.cell.2016.09.027 (2016).

20 de Almeida, R. M., Ferrari, P. F., Parmigiani, S. \& Miczek, K. A. Escalated aggressive behavior: dopamine, serotonin and GABA. Eur J Pharmacol 526, 51-64, doi:10.1016/j.ejphar.2005.10.004 (2005). 
21 Sundberg, M. et al. Improved Cell Therapy Protocols for Parkinson's Disease Based on Differentiation Efficiency and Safety of hESC-, hiPSC-, and Non-Human Primate iPSC-Derived Dopaminergic Neurons. STEM CELLS 31, 1548-1562, doi:10.1002/stem.1415 (2013). Measuring Network Activity on Micro-electrode Arrays. e54900, doi:doi:10.3791/54900 (2017).

23 Kuijlaars, J. et al. Sustained synchronized neuronal network activity in a human astrocyte coculture system. Sci Rep 6, 36529, doi:10.1038/srep36529 (2016).

24 Johnson, M. A., Weick, J. P., Pearce, R. A. \& Zhang, S. C. Functional neural development from human embryonic stem cells: accelerated synaptic activity via astrocyte coculture. J Neurosci 27, 3069-3077, doi:10.1523/JNEUROSCI.4562-06.2007 (2007).

Chen, K., Holschneider, D. P., Wu, W. H., Rebrin, I. \& Shih, J. C. A spontaneous point mutation produces monoamine oxidase A/B knock-out mice with greatly elevated monoamines and anxiety-like behavior. Journal of Biological Chemistry 279, 39645-39652, doi:10.1074/jbc.M405550200 (2004).

26 Rivero, 0 . et al. Cadherin-13, a risk gene for ADHD and comorbid disorders, impacts GABAergic function in hippocampus and cognition. Transl Psychiatry 5, e655, doi:10.1038/tp.2015.152 (2015).

27 Chailangkarn, T. et al. A human neurodevelopmental model for Williams syndrome. Nature 536, 338-343, doi:10.1038/nature19067 (2016).

28 Zoghbi, H. Y. \& Bear, M. F. Synaptic Dysfunction in Neurodevelopmental Disorders Associated with Autism and Intellectual Disabilities. Cold Spring Harbor Perspectives in Biology 4, doi:ARTN a009886 10.1101/cshperspect.a009886 (2012).

29 Zweifel, L. S. et al. Disruption of NMDAR-dependent burst firing by dopamine neurons provides selective assessment of phasic dopamine-dependent behavior. Proc Natl Acad Sci U S A 106, 7281-7288, doi:10.1073/pnas.0813415106 (2009).

30 Wu, H. F., Chen, K. \& Shih, J. C. Site-directed mutagenesis of monoamine oxidase A and B: role of cysteines. Mol Pharmacol 43, 888-893 (1993).

31 Zhang-Hooks, Y., Agarwal, A., Mishina, M. \& Bergles, D. E. NMDA Receptors Enhance Spontaneous Activity and Promote Neuronal Survival in the Developing Cochlea. Neuron 89, 337-350, doi:10.1016/j.neuron.2015.12.016 (2016).

32 Frega, M. et al. Neuronal network dysfunction in a human model for Kleefstra syndrome mediated by enhanced NMDAR signaling. bioRxiv (2019).

33 Tolias, K. F. et al. The Rac1-GEF Tiam1 couples the NMDA receptor to the activity-dependent development of dendritic arbors and spines. Neuron 45, 525-538, doi:10.1016/j.neuron.2005.01.024 (2005).

34 Ewald, R. C., Van Keuren-Jensen, K. R., Aizenman, C. D. \& Cline, H. T. Roles of NR2A and NR2B in the development of dendritic arbor morphology in vivo. I Neurosci 28, 850-861, doi:10.1523/JNEUROSCI.5078-07.2008 (2008).

35 Sepulveda, F. J. et al. Differential roles of NMDA Receptor Subtypes NR2A and NR2B in dendritic branch development and requirement of RasGRF1. J Neurophysio/ 103, 1758-1770, doi:10.1152/jn.00823.2009 (2010).

36 Soto, D., Altafaj, X., Sindreu, C. \& Bayes, A. Glutamate receptor mutations in psychiatric and neurodevelopmental disorders. Commun Integr Biol 7, e27887, doi:10.4161/cib.27887 (2014).

37 Lee, E. J., Choi, S. Y. \& Kim, E. NMDA receptor dysfunction in autism spectrum disorders. Curr Opin Pharmacol 20, 8-13, doi:10.1016/j.coph.2014.10.007 (2015).

38 Bortolato, M., Floris, G. \& Shih, J. C. From aggression to autism: new perspectives on the behavioral sequelae of monoamine oxidase deficiency. I Neural Transm (Vienna), doi:10.1007/s00702-018-1888-y (2018).

39 Sabol, S. Z., Hu, S. \& Hamer, D. A functional polymorphism in the monoamine oxidase A gene promoter. Human Genetics 103, 273-279, doi:DOI 10.1007/s004390050816 (1998). 
Caspi, A. et al. Role of genotype in the cycle of violence in maltreated children. Science 297, 851-854, doi:10.1126/science.1072290 (2002).

41 Byrd, A. L. \& Manuck, S. B. MAOA, Childhood Maltreatment, and Antisocial Behavior: Metaanalysis of a Gene-Environment Interaction. Biological Psychiatry 75, 9-17, doi:https://doi.org/10.1016/j.biopsych.2013.05.004 (2014).

42 Cohen, I. L. et al. Autism severity is associated with child and maternal MAOA genotypes. Clinical Genetics 79, 355-362, doi:10.1111/j.1399-0004.2010.01471.x (2011).

43 Cohen, I. L. et al. Association of autism severity with a monoamine oxidase A functional polymorphism. Clin Genet 64, 190-197, doi:10.1034/j.1399-0004.2003.00115.x (2003).

44 Storebo, O. J. \& Simonsen, E. The Association Between ADHD and Antisocial Personality Disorder (ASPD): A Review. Journal of Attention Disorders 20, 815-824, doi:10.1177/1087054713512150 (2016).

45 Fitzpatrick, S. E., Srivorakiat, L., Wink, L. K., Pedapati, E. V. \& Erickson, C. A. Aggression in autism spectrum disorder: presentation and treatment options. Neuropsychiatr Dis Treat 12, 1525-1538, doi:10.2147/NDT.S84585 (2016).

46 Evrard, A. et al. Altered regulation of the 5-HT system in the brain of MAO-A knock-out mice. Eur J Neurosci 15, 841-851 (2002).

47 Seo, D., Patrick, C. J. \& Kennealy, P. J. Role of Serotonin and Dopamine System Interactions in the Neurobiology of Impulsive Aggression and its Comorbidity with other Clinical Disorders. Aggress Violent Behav 13, 383-395, doi:10.1016/j.avb.2008.06.003 (2008).

48 Södersten, E. et al. A comprehensive map coupling histone modifications with gene regulation in adult dopaminergic and serotonergic neurons. Nature Communications $\mathbf{9}, 1226$, doi:10.1038/s41467-018-03538-9 (2018).

49 Lu, J. F. et al. Generation of serotonin neurons from human pluripotent stem cells. Nature Biotechnology 34, 89-94, doi:10.1038/nbt.3435 (2016).

50 Mandegar, M. A. et al. CRISPR Interference Efficiently Induces Specific and Reversible Gene Silencing in Human iPSCs. Cell Stem Cell 18, 541-553, doi:10.1016/j.stem.2016.01.022 (2016).

51 Kreitzer, F. R. et al. A robust method to derive functional neural crest cells from human pluripotent stem cells. Am J Stem Cells 2, 119-131 (2013).

52 Bologna, L. L. et al. Investigating neuronal activity by SPYCODE multi-channel data analyzer. Neural Netw 23, 685-697, doi:10.1016/j.neunet.2010.05.002 (2010).

53 Maccione, A. et al. A novel algorithm for precise identification of spikes in extracellularly recorded neuronal signals. Journal of Neuroscience Methods 177, 241-249, doi:10.1016/j.jneumeth.2008.09.026 (2009).

54 Pasquale, V., Martinoia, S. \& Chiappalone, M. A self-adapting approach for the detection of bursts and network bursts in neuronal cultures. Journal of Computational Neuroscience 29, 213-229, doi:10.1007/s10827-009-0175-1 (2010).

55 Sholl, D. A. Dendritic organization in the neurons of the visual and motor cortices of the cat. J Anat 87, 387-406 (1953). 


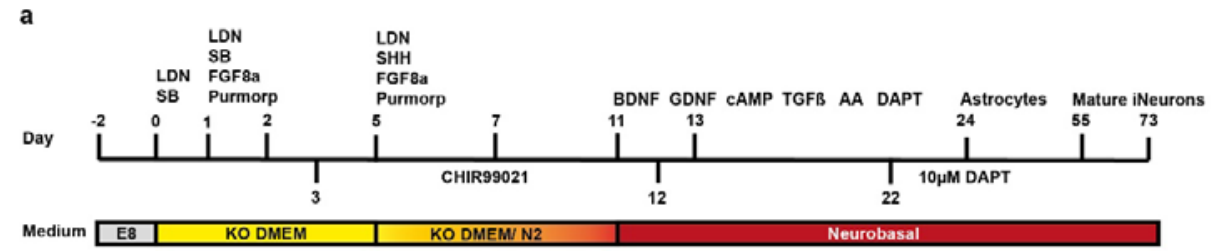

b
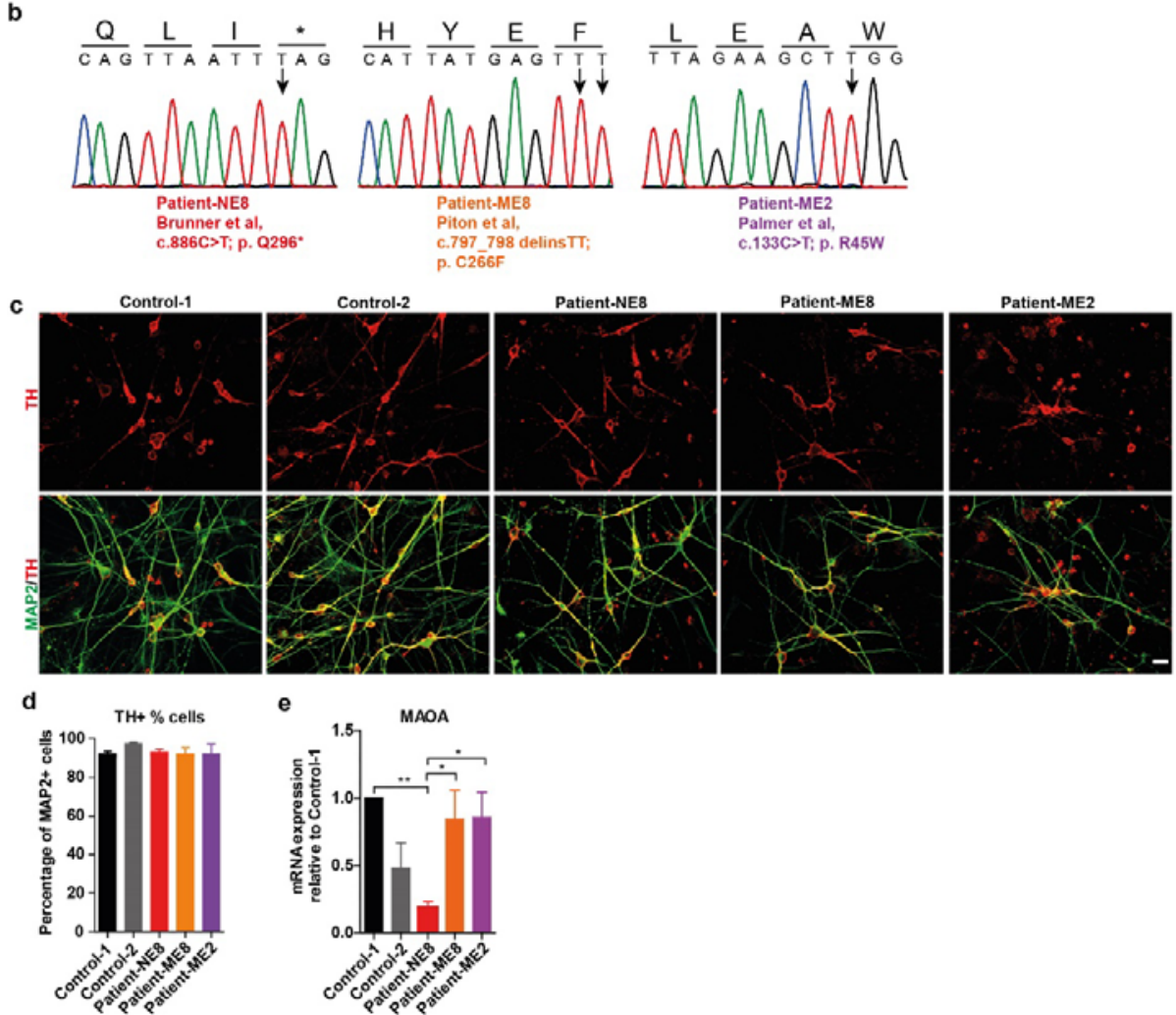

Figure 1: Differentiation of dopaminergic neurons derived from human iPSCs. (a) Schematic overview the protocol used to generate dopaminergic neurons from human iPSCs. (b) The sanger sequencing used to validate the MAOA mutations present in iPSCs lines used in this study. PatientNE8 carries a nonsense mutation at exon 8, Patient-ME2 and Patient-ME8 carry missense mutations in exon 2 and exon 8, respectively. (c) Representative images of DIV55 dopaminergic iDANs labeled by TH (red) and MAP2 (green) (Scale bar=20 $\mu \mathrm{m})$. (d) The percentage of TH-positive neurons (among MAP2-positive cells) did not differ between cell lines $(n=3)$. (e) MAOA expression in DIV73 iDANs as quantified by qPCR. MAOA mRNA expression is only significantly reduced in the patient-NE8 cell line compared to control 1 or the other patient cell lines. However, expression between patient-NE8 and control-2 was comparable. Significance was determined by one-way ANOVA followed by post-hoc Tukey's multiple comparison( $n \geq 5) .{ }^{*} p<0.05 ;{ }^{* *} p<0.01$. 
a

Control-1

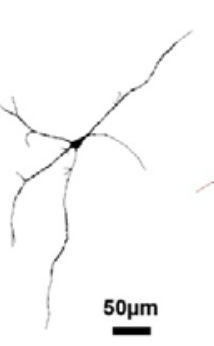

b

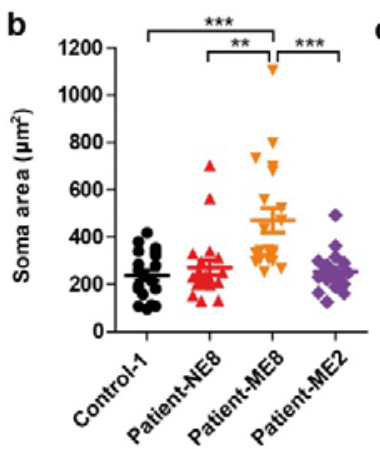

Patient-NE8
400

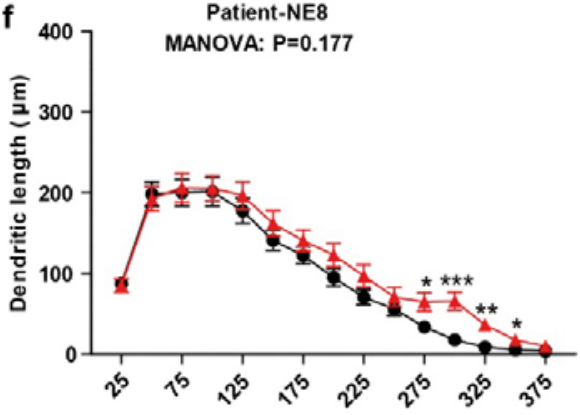

g

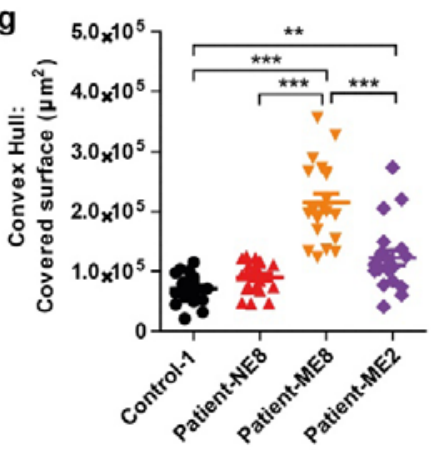

Patient-ME8

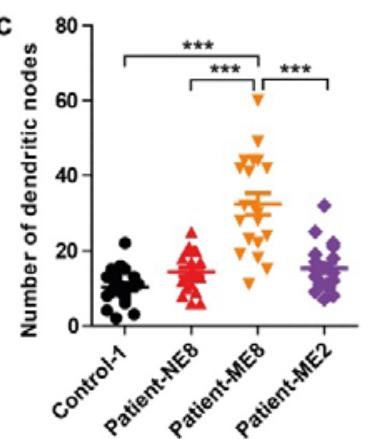

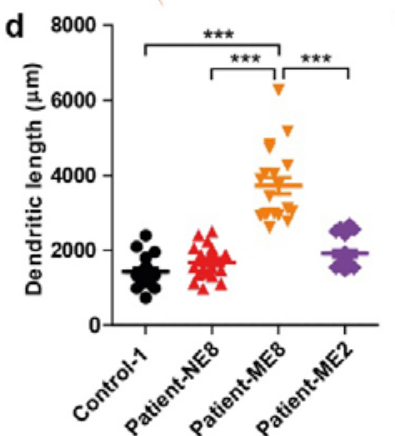

Patient-ME8

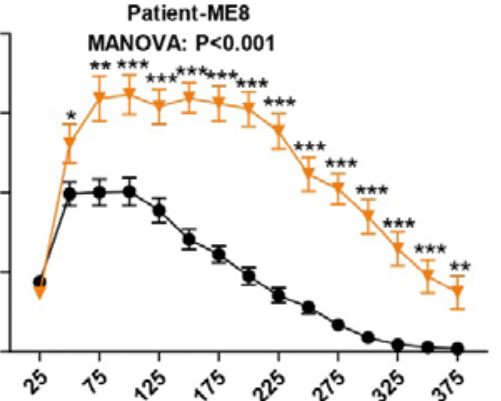

h

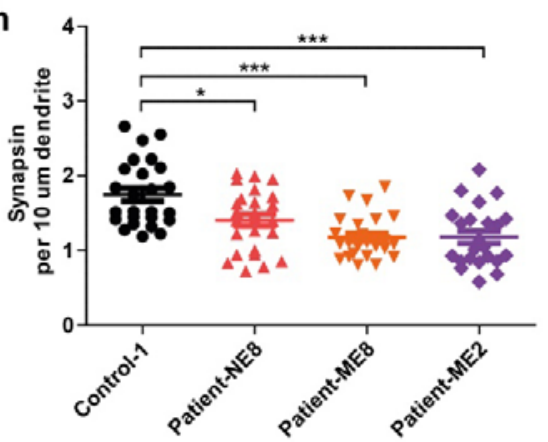

i

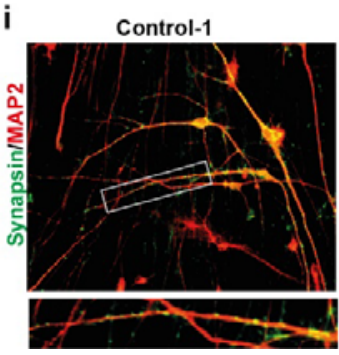

Patient-NE8

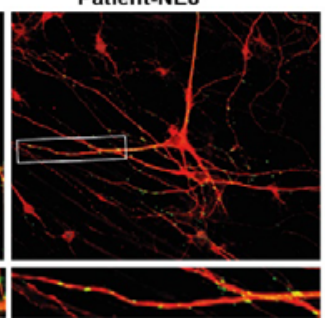

Patient-ME8

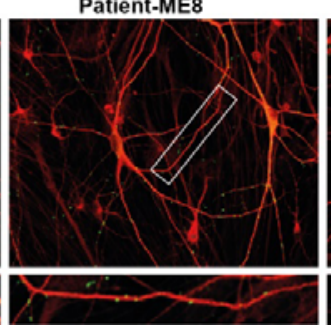

Patient-ME2

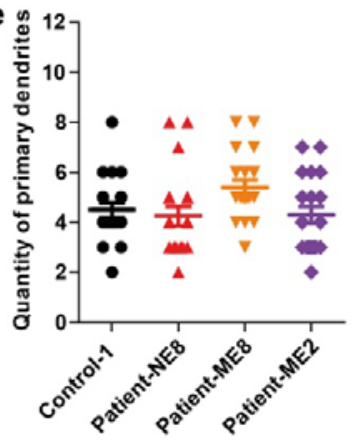

Patient-ME2

MANOVA: $P=0.096$

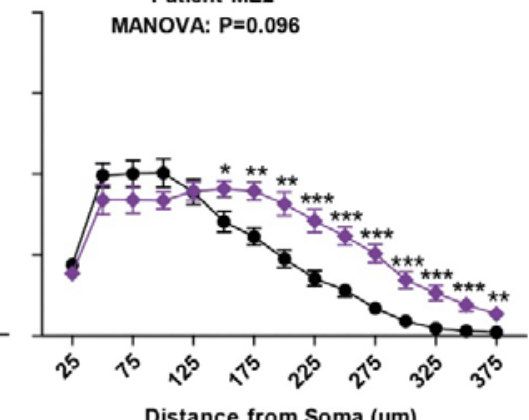

Distance from Soma ( $\mu \mathrm{m})$

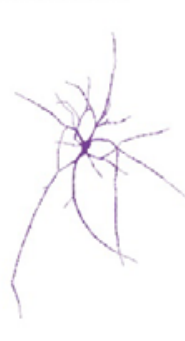


Figure 2: Morphological comparison of control and patient derived iDANs (a) Representative images of reconstructed iDANs (DIV73) iDANs $(N / n=3 / 20)$. The quantitative parameters of neuronal morphology of the day 73 iDANs were compared with the control- 1 by multivariate analysis of variance (MANOVA) in terms of soma area (b), number of dendritic nodes (c), dendritic length (d), quantity of primary dendrites (e) and Convex Hull: covered surface (g) followed by Bonferroni post-hoc correction. (f) Sholl analysis of dendritic length of DIV73 iDANs at different distances measured from soma center. Statistical analysis between groups was done using MANOVA with Wilks' lambda test. Synapsin density was decreased in the MAOA-mutant iDANs when compared with Control-1 by One-way ANOVA followed with Bonferroni post-hoc correction(h) ( $n \geq 23$ per genotype). (i) Representative images of control and patient iDANs. Dendrites are labeled with MAP2 (red) and synapses with synapsin (green), scale bar $=20 \mu \mathrm{m}$. Inset shows a single stretch of dendrite with clearly visible synapses, scale bar $=10 \mathrm{um}$. All data is represented as mean \pm SEM, asterisks indicate significant differences between genotypes. ${ }^{*} \mathrm{p}<0.05 ;{ }^{* *} \mathrm{p}<0.01 ;{ }^{* *} \mathrm{p}<0.001$. $\mathrm{N} / \mathrm{n}=$ number of batches $/$ number of neurons.
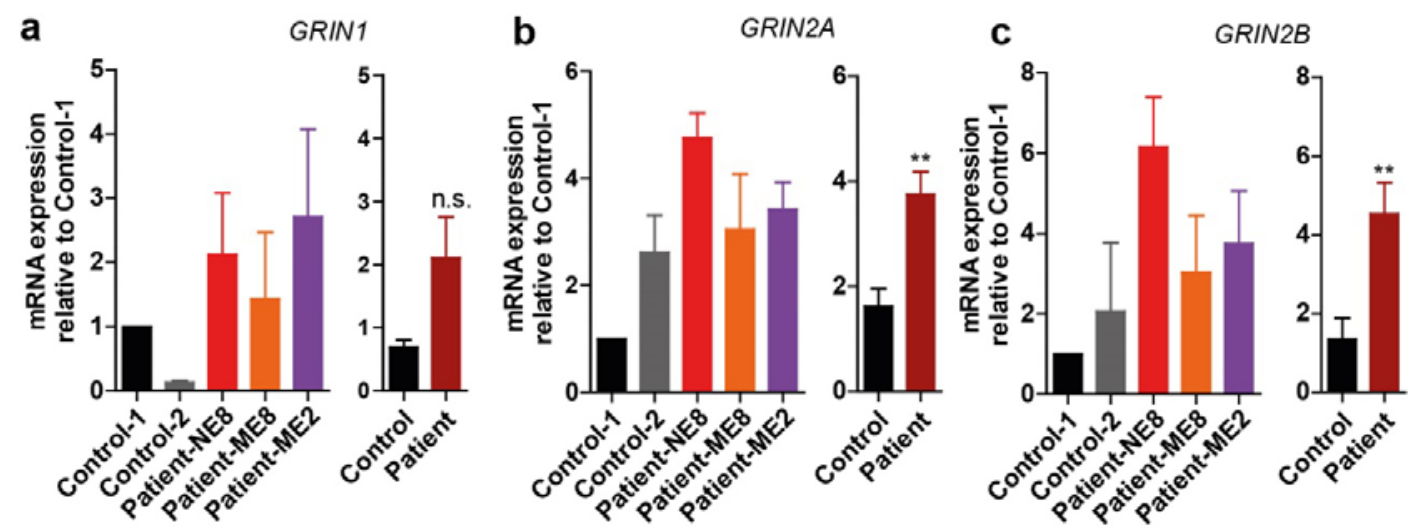

Figure 3: Patient-derived iDANs exhibit higher expression of NMDA receptor subunits GRIN2A and GRIN2B mRNA. The expression of genes encoding NMDA receptor subunits GRIN1(a), GRIN2A(b) and GRIN2B(c) was analyzed by qPCR in DIV73 iDANs. The mRNA expression was normalized to the mean of Control-1. Statistical analysis was done with Mann-Whitney U-test between 
the group of control and patient. Data are mean \pm SEM, asterisks indicate significant differences between groups. ${ }^{* *} p<0.01$. ( $\left.n \geq 5\right)$. N.s.: not significant.
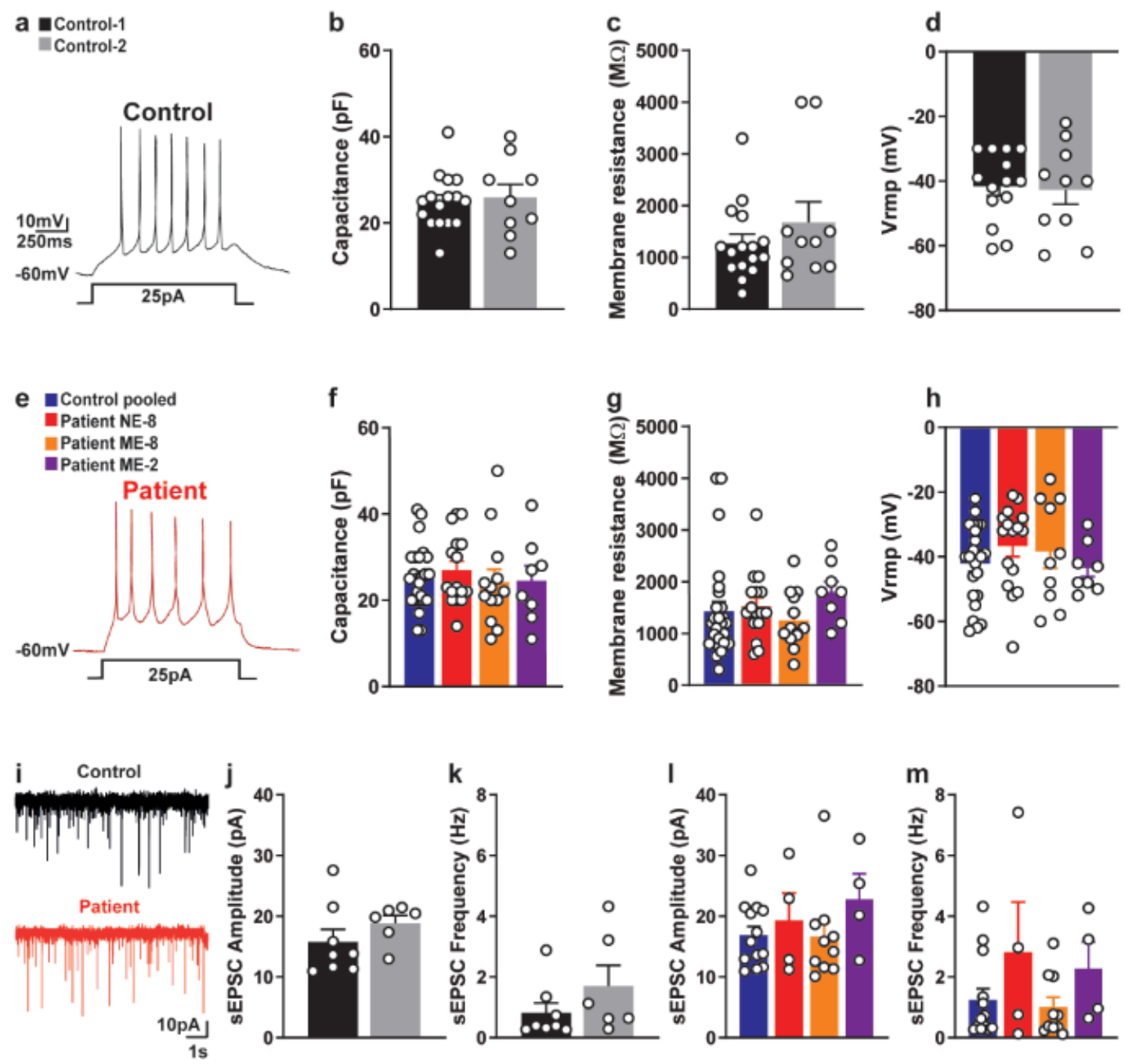

Figure 4: MAOA dysfunction does not affect intrinsic properties or sEPSC activity. (a) Representative trace of action potentials generated by DIV55 control-1 iDANs. No significant differences were observed for capacitance (b), membrane resistance (c) or resting membrane potential (d) between control-1 and control-2. (e) Representative trace of action potentials generated by DIV55 NE-8 iDANs. No differences were found between pooled control data and the patient lines, nor between the patient lines separately, in capacitance (f) membrane resistance (g) or resting membrane potential (h). (i) Representative traces of sEPSC activity in DIV73 control-1 and patientNE8 derived iDANs. sEPSC amplitude (j) and frequency ( $k$ ) were similar between control-1 and 
bioRxiv preprint doi: https://doi.org/10.1101/741108; this version posted August 21, 2019. The copyright holder for this preprint (which was not certified by peer review) is the author/funder. All rights reserved. No reuse allowed without permission.

control 2, as well as between pooled control data and patient lines $(\mathrm{l}, \mathrm{m})$. Data was analyzed by Student's T-test (control-1 vs control-2) or ANOVA (control vs patient lines) with correction for multiple testing. Data are shown as mean \pm SEM. 
a
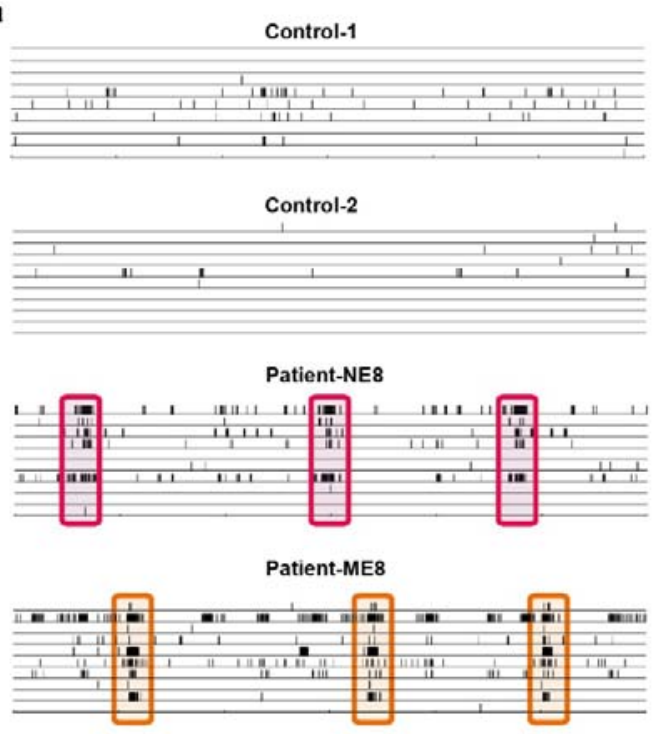

Patient-ME2

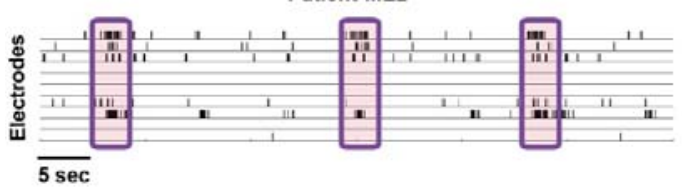

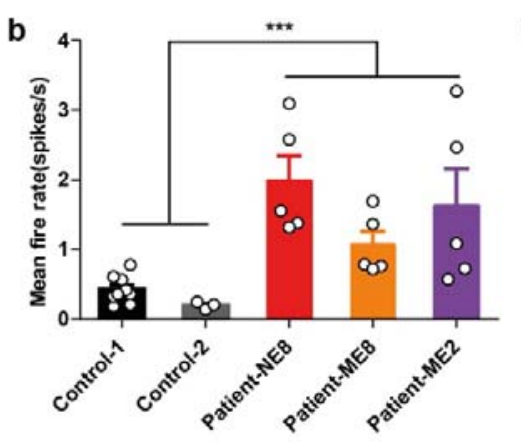

C
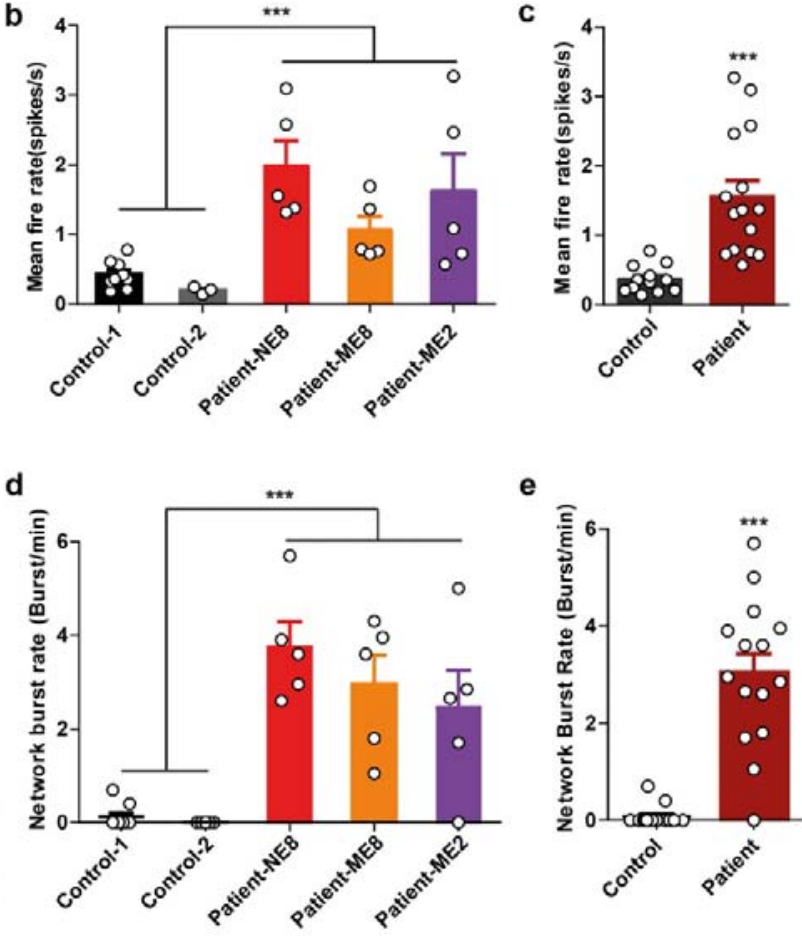

Figure 5: Increased neuronal network activity in the MAOA mutant iDANs. (a) Representative rastplot of the action potential activity across 9 micro-electrodes of day 73 iDANs. Each detected action potential is indicated as a black bar. Neuronal network burst events are highlighted by boxes in the MAOA-mutant iDANs. Both the mean fire rate, which is the average action potential frequency detected by an active electrode $(b, c)$, and the network burst rate $(\mathrm{d}, \mathrm{e})$ were increased in MAOA-mutant iDANs. Statistical analysis was performed with Mann Whitney U-test ( $n \geq 5$ per genotype). Data are mean \pm SEM; asterisks indicate significant differences between controls and MAOA-mutant iDANs. ${ }^{* * *} p<0.001$. 


\section{Supplementary material:}

a
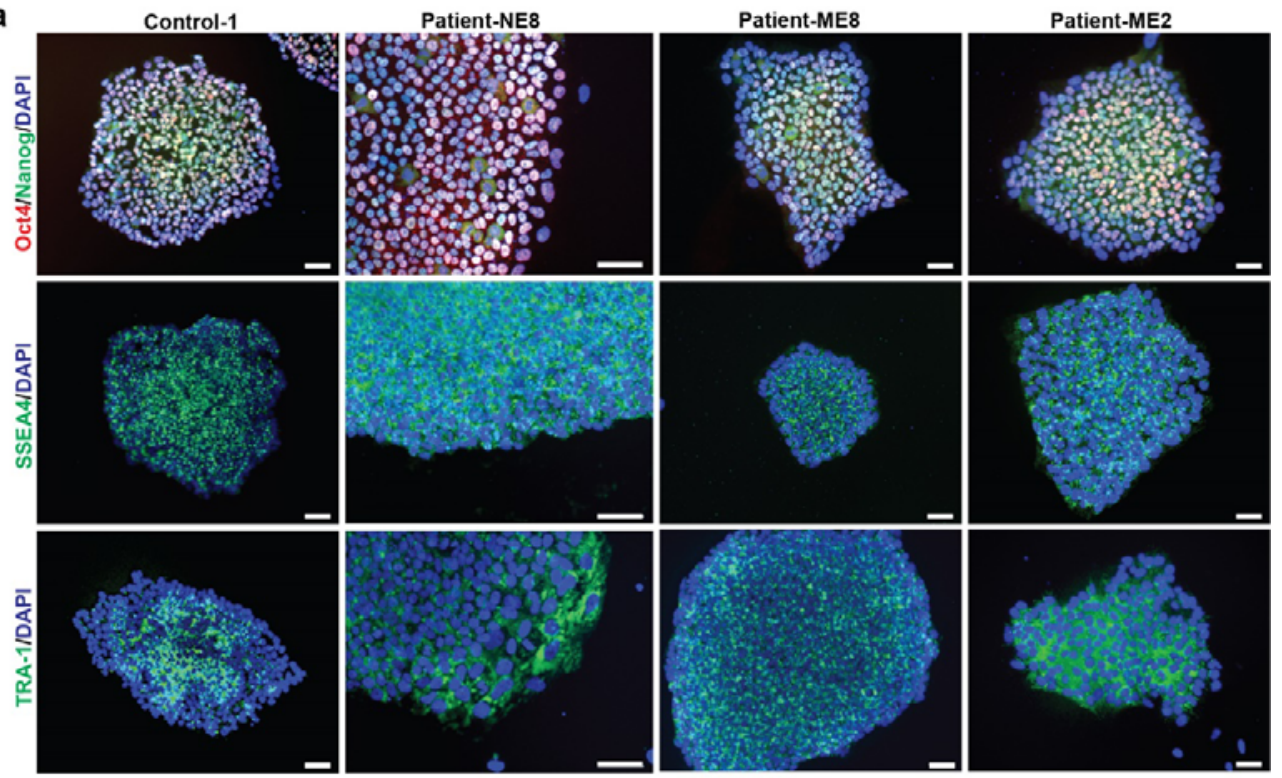

b
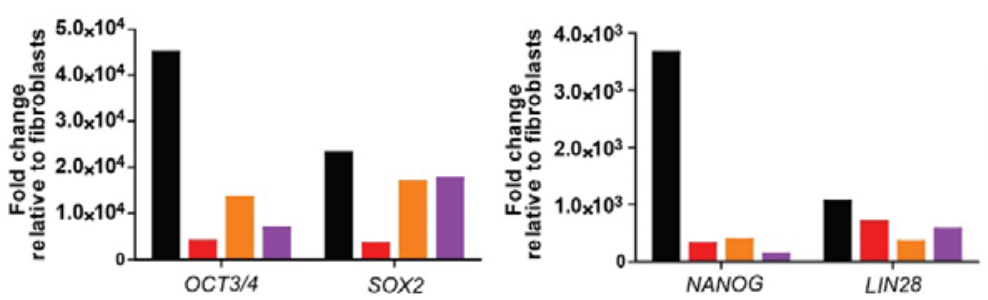
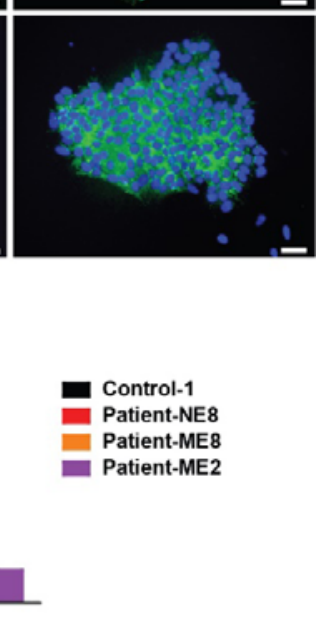

Figure S1: Characterization of iPSC lines with immunocytochemistry and qPCR. (a) All the lines were positive for the staining of pluripotent makers Oct 4, Nanog, SSEA4 and TRA-1. (b) The expression of pluripotent markers increased $10^{2}$ to $10^{5}$-folds compared to the average expression of corresponding fibroblasts (Scale bar $=50 \mu \mathrm{m})$. 


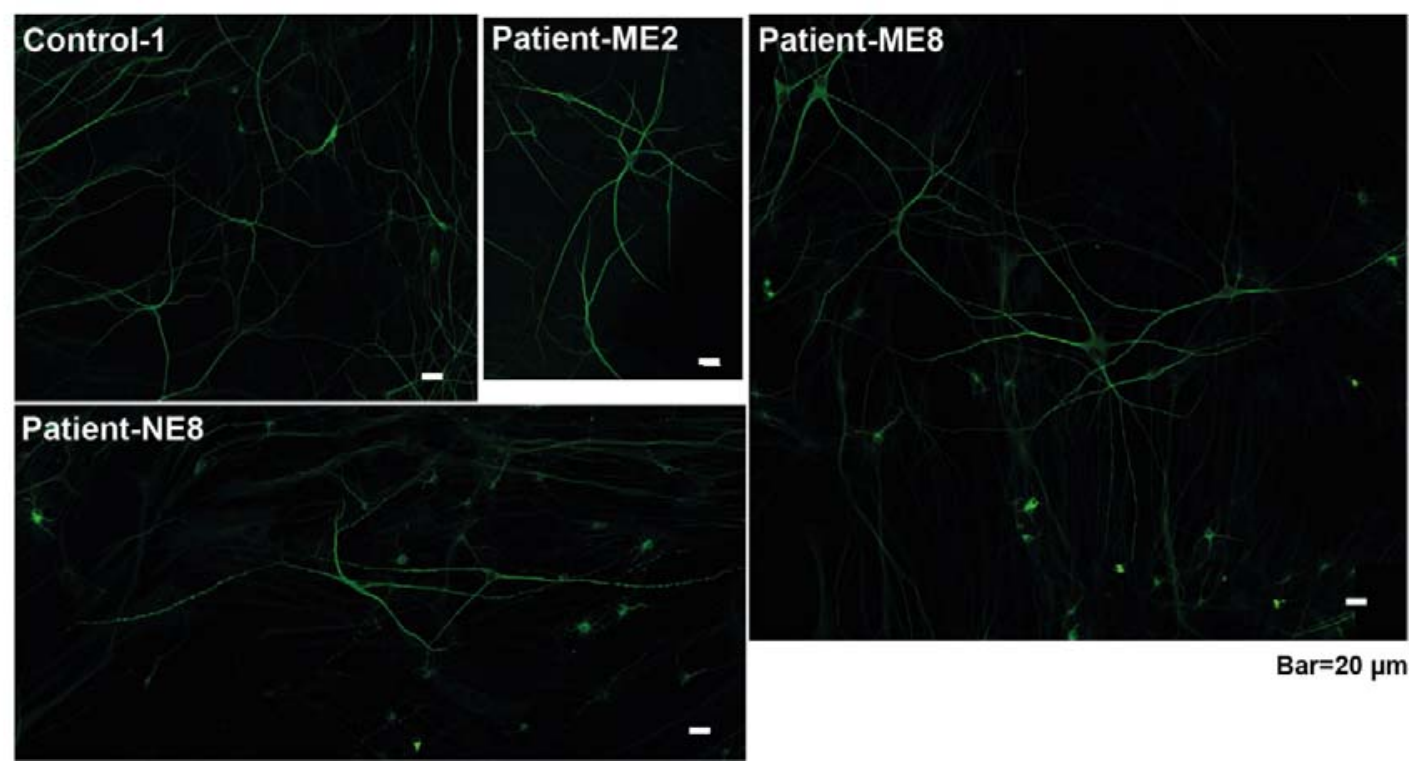

Figure S2: Representative MAP2 staining for reconstruction and Sholl analysis of neuron dendritic length. Representative MAP2 immunostaining of iDANs for the reconstruction of day 73 (Scale bar=20 $\mu \mathrm{m}$ ).
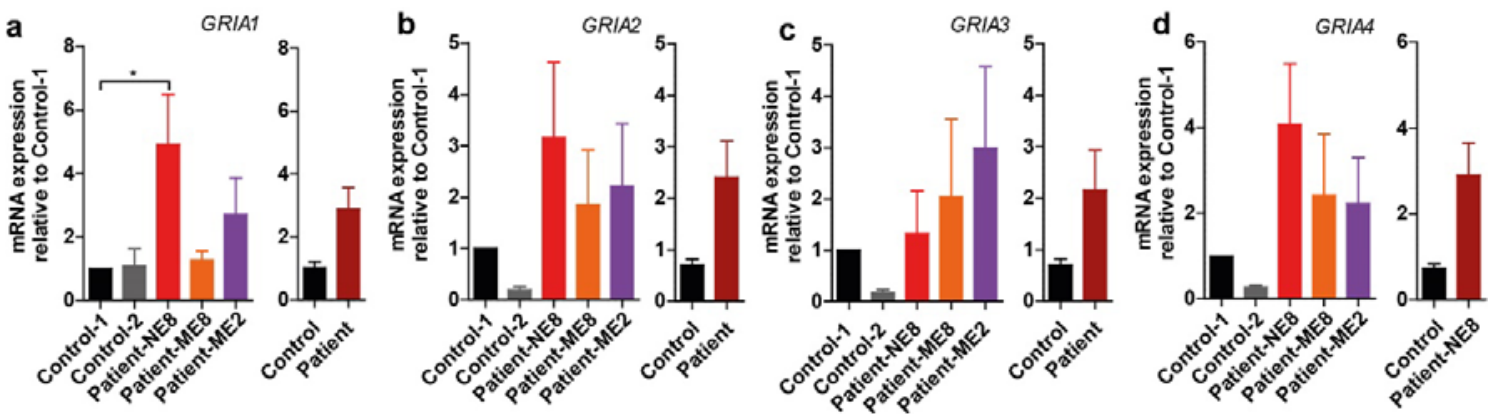

Figure S3: No significant difference of the expression of AMPA receptor subunits between control and patient. The expression of genes encoding AMPA receptor subunits GRIA1(a), GRIA2(b), GRIA3(c) and GRIA4(d) were analyzed by qPCR in day 73 iDANs. The mRNA expression were normalized to the mean of Control-1. Statistical analysis was done with Mann-Whitney U-test between the group of control and patient and one way ANOVA followed by Tukey's multiple comparison test for different genotypes. Data are mean \pm SEM, asterisks indicate significant differences between genotypes. ${ }^{*} p<0.05$. $(n \geq 5)$. 


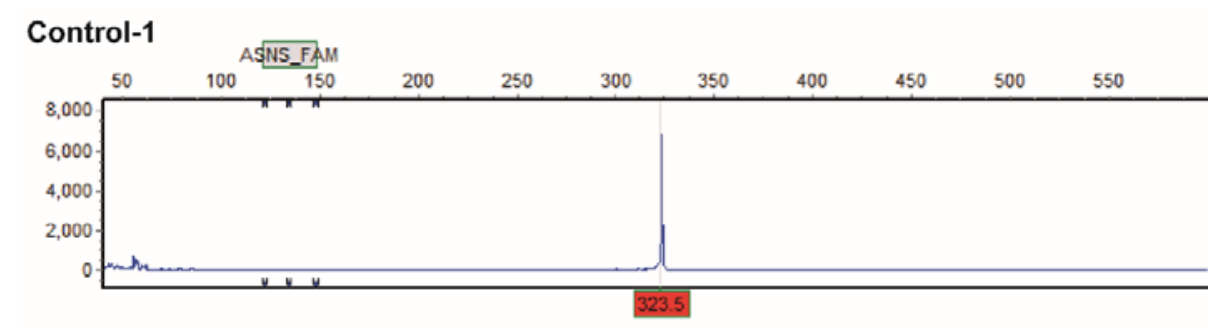

Control-2

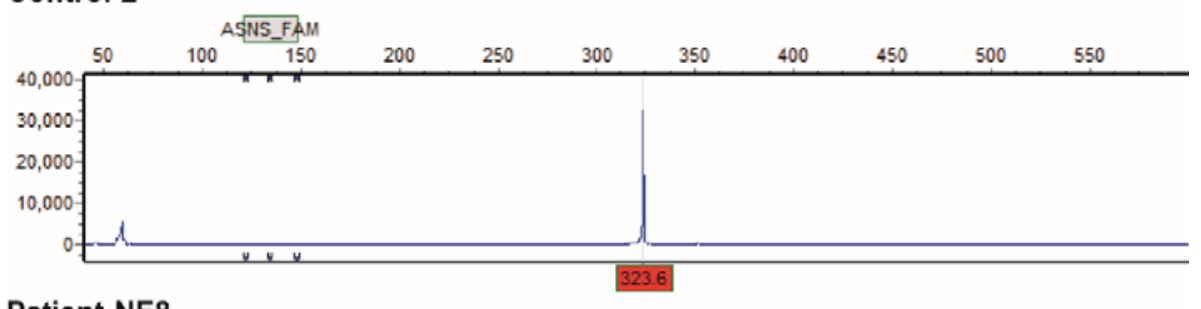

Patient-NE8

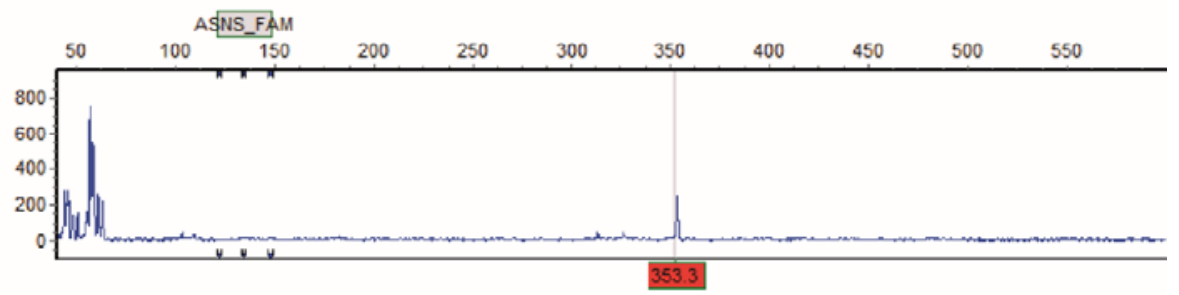

Patient-ME8

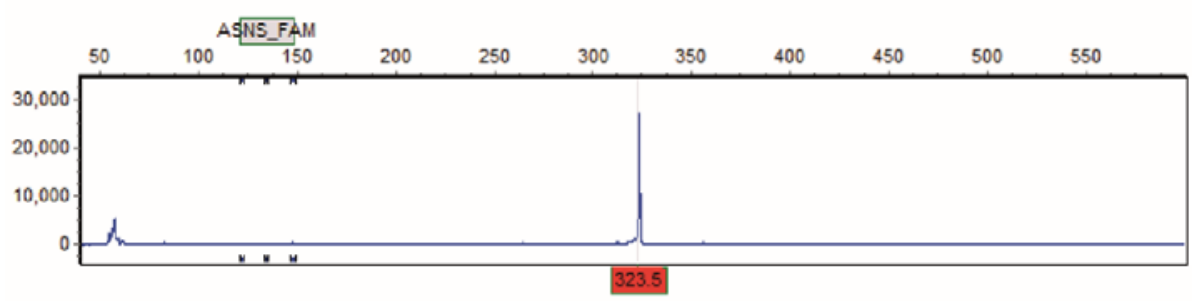

Patient-ME2

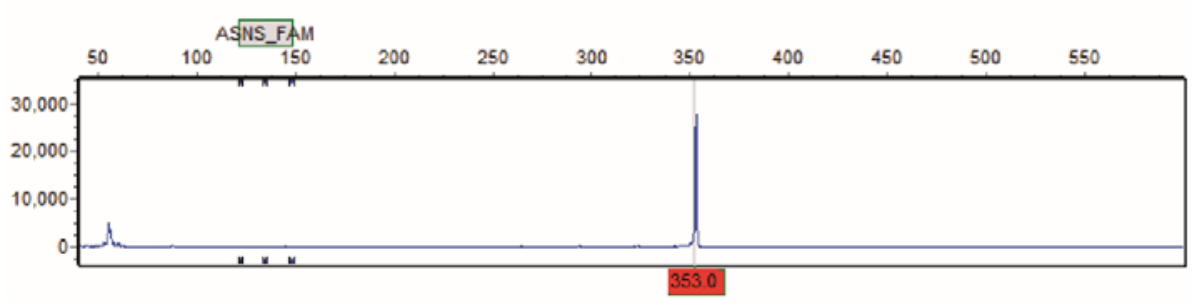

Figure S4: Variable number tandem repeats (VNTRs) polymorphism of MAOA promoter.(Corresponding to table S2). The peaks at 323 position indicate the individuals have a $3 \mathrm{R}$ allele and the peaks at 353 position indicate the individuals carry a $4 \mathrm{R}$ allele. 
Supplementary table S1: primers for qRT-PCR

\begin{tabular}{|c|c|c|}
\hline Name & Sequence & References \\
\hline B2M Forward & 5'-GCCGTGTGAACCATGTGACT-3' & 1 \\
\hline B2M Reverse & 5'-GCTTACATGTCTCGATCCCACTT-3' & \\
\hline MAOA Forward & 5'-CCTTGACTGCCAAGATTCACTTC-3' & 2 \\
\hline MAOA Reverse & 5'-TGCACTTAATGACAGCTCCCAT-3' & \\
\hline GRIN1 Forward & 5'-CGCCGCTAACCATAAACAAC-3' & 3 \\
\hline GRIN1 Reverse & 5'-GGGGAATCTCCTTCTTGACC-3' & \\
\hline GRIN2A Forward & 5'-AGCTGCTACGGGCAGATG-3' & \\
\hline GRIN2A Reverse & 5'-CCTGGTAGCCTTCCTCAGTG-3' & \\
\hline GRIN2B Forward & 5'-GGAGTTCTGGTTCCTACTGGG-3' & \\
\hline GRIN2B Reverse & 5'-TCTCATGGGAACAGGAATGG-3' & \\
\hline GRIA1 Forward & 5'-GCAGCAGTGGAAGAATAGTGATG-3' & \\
\hline GRIA1 Reverse & 5'-ATCACCTTCACCCCATCGTA-3' & \\
\hline GRIA2 Forward & 5'-GCTTGGTGCTAAATTGCTGT-3' & \\
\hline GRIA2 Reverse & 5'-TCCAAGAAAAGTAGAGCATCCA-3' & \\
\hline GRIA3 Forward & 5'-TTCCCACTGGAGGCATGTG-3' & \\
\hline GRIA3 Reverse & 5'-CATCAGCAATATTCGTGTCATGC-3' & \\
\hline GRIA4 Forward & 5'-TGCTGCAACTAAGACCTTCGTTAC-3' & \\
\hline GRIA4 Reverse & 5'-TCGAGTATCCCCTGTCTGTGTC-3' & \\
\hline
\end{tabular}


Supplementary table S2: information about the cell lines used in the study

\begin{tabular}{|l|l|l|l|l|l|}
\hline Cell lines & Gender & $\begin{array}{l}\text { Age of } \\
\text { biopsy(year) }\end{array}$ & $\begin{array}{l}\text { MAOA } \\
\text { Mutation }\end{array}$ & MAOA \\
polymorphism & VNTR & Reference \\
\hline Control-1 & Male & 9 & none & 3R, low expression allele & NA \\
\hline Control-2 & Male & NA & none & 3R, low expression allele & 4 \\
\hline Patient-NE8 & Male & 21 & $\begin{array}{l}\text { c.886C>T; } \\
\text { p.Q296* }\end{array}$ & 4R, high expression allele & 5 \\
\hline Patient-ME8 & Male & 10 & $\begin{array}{l}\text { c.797_798 } \\
\text { delinsTT; p. }\end{array}$ & 3R, low expression allele & 6 \\
& & & C266F & & \\
\hline Patient-ME2 & Male & 16 & c.133C>T; & 4R, high expression allele & 7 \\
& & & p.R45W & & \\
\hline
\end{tabular}

NA: not available

\section{Supplementary methods:}

Genotyping of the MAOA promoter VNTR polymorphism: The genomic DNA was amplified with $1 \mathrm{x}$ AmpliTaq Gold® 360 Master Mix (Life Technologies) and $0.33 \mathrm{mM}$ of fluorescently labeled forward primer (FAM-5'-ACAGCCTGACCGTGGAGAAG-3') and reverse primer (5'GAACGGACGCTCCATTCGGA-3') in a total volume of $7,5 \mu \mathrm{l}$ using the protocol: $95^{\circ} \mathrm{C}$ for $10 \mathrm{~min}$ followed by 35 cycles of denaturation for $30 \mathrm{~s}$ at $95^{\circ} \mathrm{C}, 30 \mathrm{~s}$ annealing at $60^{\circ} \mathrm{C}$, primer extension at $72^{\circ} \mathrm{C}$ for $1 \mathrm{~min}$ and a final extension at $72^{\circ} \mathrm{C}$ for $10 \mathrm{~min}$. Fragment length analysis of the PCR product was performed by an automated capillary sequencer ABI3730 (Applied Biosystems, Nieuwerkerk a/d ljssel, The Netherlands) using standard conditions (1 ul of the 1:20 diluted PCR product together with 9.7 ul formamide and 0.3ul GeneScan-600 Liz Size Standard TM (Applied Biosystems, Nieuwekerk aan den ljsel, the Netherlands). Results were analyzed with GeneMarker version 2.6.7 (SoftGenetics, US).

\section{References:}


1 Sanchez-Danes, A. et al. Efficient generation of A9 midbrain dopaminergic neurons by lentiviral delivery of $L M X 1 A$ in human embryonic stem cells and induced pluripotent stem cells. Human gene therapy 23, 56-69, doi:10.1089/hum.2011.054 (2012).

$2 \mathrm{Li}, \mathrm{J}$. et al. Monoamine oxidase A suppresses hepatocellular carcinoma metastasis by inhibiting the adrenergic system and its transactivation of EGFR signaling. Journal of hepatology 60, 1225-1234, doi:10.1016/j.jhep.2014.02.025 (2014).

3 Frega, M. et al. Neuronal network dysfunction in a human model for Kleefstra syndrome mediated by enhanced NMDAR signaling. bioRxiv (2019).

4 Mandegar, M. A. et al. CRISPR Interference Efficiently Induces Specific and Reversible Gene Silencing in Human iPSCs. Cell Stem Cell 18, 541-553, doi:10.1016/j.stem.2016.01.022 (2016).

5 Brunner, H. G., Nelen, M., Breakefield, X. O., Ropers, H. H. \& van Oost, B. A. Abnormal behavior associated with a point mutation in the structural gene for monoamine oxidase $A$. Science 262, 578-580 (1993).

6 Piton, A. et al. 20 ans apres: a second mutation in MAOA identified by targeted highthroughput sequencing in a family with altered behavior and cognition. Eur J Hum Genet 22, 776-783, doi:10.1038/ejhg.2013.243 (2014).

7 Palmer, E. E. et al. New insights into Brunner syndrome and potential for targeted therapy. Clinical Genetics 89, 120-127, doi:10.1111/cge.12589 (2016). 